\title{
Serial Noninvasive Targeted Imaging of Peripheral Angiogenesis: Validation and Application of a Semiautomated Quantitative Approach
}

Lawrence W. Dobrucki ${ }^{1}$, Donald P. Dione ${ }^{1}$, Leszek Kalinowski ${ }^{2}$, Donna Dione ${ }^{1}$, Marivi Mendizabal ${ }^{3}$, Jun $\mathrm{Yu}^{4}$, Xenophon Papademetris ${ }^{5}$, William C. Sessa ${ }^{4}$, and Albert J. Sinusas ${ }^{1,5}$

${ }^{I}$ Section of Cardiovascular Medicine, Department of Internal Medicine, Yale University School of Medicine, New Haven, Connecticut; ${ }^{2}$ Department of Clinical Chemistry and Biochemistry, Medical University of Gdansk, Gdansk, Poland; ${ }^{3} G E$ Healthcare, Buckinghamshire, United Kingdom; ${ }^{4}$ Department of Pharmacology, Yale University School of Medicine, New Haven, Connecticut; and ${ }^{5}$ Department of Diagnostic Radiology, Yale University School of Medicine, New Haven, Connecticut

Previous studies by our group have demonstrated the feasibility of noninvasive imaging of $\alpha_{v}$ integrin to assess temporal and spatial changes in peripheral and myocardial angiogenesis. In this study, we validate the reproducibility, accuracy, and applicability of a new semiautomated noninvasive approach for serial quantitative evaluation of targeted micro-SPECT/CT images of peripheral angiogenesis in wild-type and endothelial nitric oxide sythase (eNOS)-deficient (eNOS-/-) mice subjected to hindlimb ischemia. Methods: Mice $(n=15)$ underwent surgical ligation of the right femoral artery to induce unilateral hindlimb ischemia. One week after ligation, a ${ }^{99 m}$ Tc-labeled cyclic-ArgGly-Asp peptide targeted at $\alpha_{v}$ integrin (NC100692, $n=10$ ) or a ${ }^{99 m}$ Tc-labeled negative control $(\mathrm{AH}-111744, n=5)$ was injected, and $60 \mathrm{~min}$ later in vivo micro-SPECT/CT images were acquired. Mice were euthanized, tissue from proximal and distal hindlimb was excised for $\gamma$-well counting (GWC) of radiotracer activity, and ischemic-to-nonischemic (I/NI) ratio was calculated. Micro-SPECT/CT images were analyzed using a new semiautomated approach that applies complex volumes of interest (VOIs) derived from segmentation of the micro-CT images onto microSPECT images to calculate $\mathrm{I} / \mathrm{NI}$ activity ratios for the proximal and distal hindlimb. Studies were reprocessed for determination of intra- and interobserver variability. To compare 3-dimensional (3D) VOI analysis with traditional manual 2-dimensional regionof-interest (ROI) analysis of maximum-intensity-projection images, micro-SPECT images were summed onto a single anterior-posterior projection. Rectangular ROls were manually drawn and I/NI ratio calculated. Our new 3D analysis approach was applied to additional groups of mice (eNOS $-/-, n=5$; wild-type, $n=3$ ) imaged before and 1 and 4 wk after femoral artery resection. Results: Our new semiautomated approach for the evaluation of images of $\alpha_{v}$ integrin targeted with microSPECT/CT demonstrated both a high intra- and interobserver variability $\left(R^{2}=0.997\right)$ and an accuracy $\left(R^{2}=0.780\right)$ for estimation of relative radiotracer activity relative to GWC. Analysis of se-

Received Dec. 2, 2008; revision accepted Apr. 15, 2009.

For correspondence or reprints contact: Albert J. Sinusas, Section of Cardiovascular Medicine, Yale University School of Medicine, P.O. Box 208017, 3FMP New Haven, CT 06520-8017.

E-mail: albert.sinusas@yale.edu

COPYRIGHT $\odot 2009$ by the Society of Nuclear Medicine, Inc. rial micro-SPECT/CT images demonstrated a significant increase in relative NC100692 retention in the ischemic hindlimb of both wild-type and eNOS-/- mice at 1 wk after surgery. There was a significant $(\sim 25 \%)$ decrease in radiotracer uptake in eNOS $-/-$ mice relative to wild-type animals, which was not observed at baseline or 4 wk after ligation. Conclusion: A new semiautomated analysis of images of $\alpha_{v}$ integrin targeted with micro-SPECT/CT provides a noninvasive approach for serial quantitative evaluation of peripheral angiogenesis. The reproducibility and accuracy of this approach allows for quantitative analysis of serial targeted molecular images of lower extremities, has applicability to other targeted SPECT or PET radiotracers, and may have implications for clinical imaging in patients with peripheral arterial disease.

Key Words: molecular imaging; micro-SPECT; micro-CT; peripheral angiogenesis

J Nucl Med 2009; 50:1356-1363

DOI: 10.2967/jnumed.108.060822

$\mathbf{T}$ he introduction of genetic and cell-based therapies and nanoparticle drug-delivery systems for the treatment of cardiovascular diseases through stimulation of angiogenesis has motivated the development of noninvasive imaging approaches to evaluate and track the response to these therapies. Angiogenesis is a complex process that involves many stimuli, growth factors, and interactions between multiple cell types. Several experimental models have been developed to evaluate this process and novel therapeutic interventions in normal and transgenic mice. The experimental evaluation of peripheral angiogenesis traditionally has relied on the assessment of the pathologic and physiologic changes associated with the angiogenic process, using well-established models of hindlimb ischemia to define the molecular events associated with angiogenesis. The genetic loss of endothelial nitric oxide sythase (eNOS) was found in models of hindlimb ischemia to impair vascular endothelial growth factor signaling, ischemia- 
induced angiogenesis, and blood flow recovery and was associated with limb necrosis (1). These mechanistic studies of the angiogenic process generally require the euthanasia of animals at selected time points because of the application of highly invasive measures or tissue analyses. Several investigators have demonstrated the feasibility of noninvasively evaluating angiogenesis using image-based approaches targeted at cell surface receptors that are upregulated or activated in association with the angiogenic process (2-7).

Peptides containing the tripeptide sequence Arg-Gly-Asp (RGD) are known to bind with a high affinity to the $\alpha_{\mathrm{v}}$ integrins associated with angiogenesis. Investigators have reported the use of radiolabeled tracers containing RGD motif for the noninvasive imaging of angiogenesis in different animal models $(8-11)$. A ${ }^{99 \mathrm{~m} T c-l a b e l e d ~ c y c l i c ~ R G D ~ p e p t i d e ~}$ ( ${ }^{99 \mathrm{~m} T c-N C 100692 ; ~ G E ~ H e a l t h c a r e) ~ t a r g e t e d ~ a t ~} \alpha_{\mathrm{v}}$ integrins was demonstrated to provide efficacy and safety of imaging malignant breast tumors (8). More recently, Edwards et al. demonstrated that NC100692 had a high affinity for the $\alpha_{\mathrm{v}}$ integrins, was metabolically stable, and had a biodistribution favorable for in vivo imaging purposes (9).

We previously demonstrated the potential of planar $\gamma$-camera imaging with ${ }^{99 \mathrm{~m} T c-N C 100692}$ for the evaluation of spatial and temporal changes in peripheral angiogenesis in mice subjected to hindlimb ischemia (5). This previous study demonstrated a positive correlation between quantitative analysis of planar images of NC100692 and $\gamma$-well counting (GWC) of tissue specimens, although the analysis of in vivo planar pinhole images tended to underestimate the magnitude of the relative increases in NC100692 retention within the ischemic hindlimb. We hypothesized that these differences might be due to attenuation and partial-volume errors and the selection of inappropriate regions of interest (ROIs) and that images could be more accurately quantified by the use of a high-resolution hybrid micro-SPECT/CT system that would provide anatomic localization of the targeted radiotracer within the intended tissues.

In the current study, we describe a new noninvasive approach for the serial quantitative evaluation of radiotracers targeted at $\alpha_{\mathrm{v}}$ integrins in established models of hindlimb ischemia for the evaluation of ischemia-induced angiogenesis. Initial studies were performed to develop and validate the accuracy and reproducibility of a semiautomated imageanalysis approach. Additional studies were performed to noninvasively define the temporal changes in angiogenesis in control mice and eNOS-knockout (eNOS-l-) mice in response to ischemic injury using our quantitative hybrid micro-SPECT/CT $\alpha_{\mathrm{v}}$ integrin-targeted imaging approach. Quantitative analysis of micro-SPECT/CT images of $\alpha_{\mathrm{v}}$ integrins and other targeted biologic markers will be critical for understanding the angiogenic process and tracking novel molecular or genetic therapies applied to models of hindlimb ischemia and may have implications for the evaluation of patients with peripheral arterial disease.

The method proposed here can be applied to quantify SPECT/CT or PET/CT images of virtually any radiotracer within limbs of animals or patients and represents an important step in standardizing the quantification of nuclear images of the peripheral limbs.

\section{MATERIALS AND METHODS}

Overview of Surgical Model and Experimental Protocol

All experiments were performed according to the guiding principles of the American Physiologic Society and approved by the Institutional Animal Care and Use Committee. Male C57BL/6 mice (Charles River Laboratories) were anesthetized with 1\%-3\% isoflurane for surgical intervention and imaging. For initial methodologic development and validation of the $\alpha_{\mathrm{v}}$ integrin-targeted micro-SPECT/CT approach, a subset of mice $(n=15)$ underwent sterile surgical occlusion of the right femoral artery to induce unilateral hindlimb ischemia according to the modified procedure described previously (5). The surgical model used in the initial validation studies resulted in a small postoperative wound with minimal inflammation. These mice underwent $\alpha_{v}$ integrin-targeted micro-SPECT/CT at $7 \mathrm{~d}$ after surgery, a time point previously established to correspond with maximal activation of the $\alpha_{\mathrm{v}}$ integrins (5). For further application of the developed quantitative approach for the noninvasive serial imaging of angiogenesis, additional wild-type (eNOS $+/+, n=3)$ and eNOS $-/-$ mice $(n=5)$ underwent sterile surgical removal of the left femoral artery to induce severe unilateral hindlimb ischemia, using a surgical approach previously described (1). Before each imaging session, a PE10 catheter (Becton Dickinson) was placed into the right jugular vein for radiotracer injections.

\section{Micro-SPECT/CT Protocol}

For the validation studies, all animals $(n=15)$ were injected intravenously with approximately $37 \mathrm{MBq}$ of either the ${ }^{99 \mathrm{~m}} \mathrm{Tc}-$ labeled chelate-peptide conjugate containing an RGD motif $\left({ }^{99 \mathrm{~m}} \mathrm{Tc}-\right.$ NC100692, $n=10)$ targeted at the $\alpha_{\mathrm{v}}$ integrin or a negative-control scrambled peptide ( $\left.{ }^{99 \mathrm{~m}} \mathrm{Tc}-\mathrm{AH}-111744-01, n=5\right) 7 \mathrm{~d}$ after surgical induction of hindlimb ischemia. Imaging was performed with a hybrid dual-head micro-SPECT/CT small-animal scanner (X-SPECT; GammaMedica-Ideas) equipped with 1-mm pinhole collimators. Animals were placed supine on the bed with legs secured in an extended position. Sixty minutes after radiotracer injection, mice underwent SPECT (32 projections, $60 \mathrm{~s} /$ projection per head) with a $20 \%$ energy window centered at $140 \mathrm{keV}$, followed by highresolution anatomic CT (512 projections, $50 \mathrm{kVp} / 600 \mu \mathrm{A}$ energy). Mice were euthanized immediately after CT was completed, and soft-tissue samples from both legs were taken for GWC. For serial imaging studies, animals $(n=8)$ were injected intravenously with approximately $37 \mathrm{MBq}$ of ${ }^{99 \mathrm{~m}} \mathrm{Tc}-\mathrm{NC} 100692$ before and after 1 and 4 wk of hindlimb ischemia and imaged according to the protocol described above.

\section{Micro-SPECT/CT Image Reconstruction and Analysis: Validation Studies}

Image Reconstruction and Analysis. The micro-SPECT and micro-CT images were reconstructed using existing commercial software (X-Flex; GammaMedica-Ideas and Cobra Exim, respectively). Micro-SPECT images were filtered (Butterworth; cutoff, $0.5 \times$ Nyquist frequency; order of 6 ), fused with micro-CT images and converted to Analyze format (Mayo Foundation). The relative uptake of targeted tracer within the soft tissue of the hindlimbs was quantified using semiautomated software developed as part of the current study and now available online (http://www. 
bioimagesuite.org/). Complex irregular volumes of interest (VOIs) were generated from the micro-CT images as described below and registered with the micro-SPECT images. These complex regions included only soft-tissue structures from the hindlimb with skeletal structures removed from the image during the segmentation process.

To compare our semiautomatic volumetric quantification of 3-dimensional (3D) micro-SPECT images with more traditional 2-dimensional (2D) analysis of planar or maximum-intensityprojection nuclear images, micro-SPECT images were summed onto a single anterior-posterior projection, and rectangular $2 \mathrm{D}$ ROIs were manually drawn on the maximum-intensity-projection image. The mean counts from each $2 \mathrm{D}$ region of interest (ROI) within ischemic hindlimb were calculated and normalized to the contralateral ROIs drawn on nonischemic hindlimb and expressed as an ischemic-to-nonischemic (I/NI) ratio.

For 3D volumetric image analysis, micro-CT images were loaded into our image-analysis tool and visualized as a semitranslucent 3D rendering. The micro-CT image intensity was adjusted by the operator to optimally visualize the skeletal and muscular structures. The operator positioned 8 planes ( 4 on each side) to segment the micro-CT image of a mouse into 7 regions including the main thorax with the tail, left proximal and distal hindlimb, left foot, right proximal and distal hindlimb, and right foot. Two planes were used to define each hip joint, 1 axial above the femur and 1 sagittal at the pelvic joint. The knee was axially bisected between the femur and tibia. A final plane was used at the ankle joint to separate the foot. After the planes were positioned, automatic K-means clusteringbased segmentation removed the bone from the micro-CT image. A 7-region segmentation image or object map (an integer-valued image in the range $0-6$ in which the labels $1-7$ were used to identify the 7 regions) was automatically created, excluding the skeleton from the VOIs (Fig. 1). This object map was then applied to the corresponding micro-SPECT images to determine mean counts in each VOI. The radiotracer activity from the ischemic proximal and distal hindlimb was normalized by the contralateral nonischemic proximal and distal hindlimb, respectively, to compute I/NI ratio.

Image Analysis Versus GWC. To validate the accuracy of the quantitative noninvasive targeted-imaging approach, imagederived results from our new 3D VOI and traditional 2D ROI analysis were compared with GWC of the corresponding tissue samples. The soft tissues in hindlimbs were excised, excluding the bones, and divided into proximal and distal sections according to the position of the major joints. The tissue samples were weighed, and the ${ }^{99 m}$ Tc activity was measured using a $\gamma$-well counter (Cobra Packard) and corrected for background, decay time, and tissue weight. The corrected counts from the proximal and the distal hindlimbs were used to calculate the I/NI ratios for each mouse. The registered micro-SPECT and micro-CT images and CT-derived VOIs were qualitatively inspected for background activity that might have scattered into the VOIs from adjacent nonmuscular structures, such as the bladder.

Intra- and Interobserver Variability. The intra- and interobserver variability was assessed to determine the reproducibility of this quantitative analysis. For intraobserver variability, fused microSPECT/CT image sets were analyzed twice by a single operator. The time interval between the 2 reading studies varied between a few hours and a couple of days. To define the interobserver reproducibility of the analyses, fused micro-SPECT/CT image sets were analyzed by 2 different operators. The results were expressed as I/NI ratio in distal hindlimb and statistically analyzed using a paired Student $t$ test. For the correlation between different analyses, the Pearson correlation coefficients were calculated.

\section{Serial Micro-SPECT/CT of Peripheral Angiogenesis: Application Studies}

The image-analysis approach was applied to an additional series of images acquired before and after femoral artery occlusion, to monitor peripheral angiogenesis in wild-type and eNOS-/mice subjected to severe hindlimb ischemia. The serial microSPECT and micro-CT images acquired at baseline and 1 and $4 \mathrm{wk}$ after femoral occlusion were reconstructed, fused, and converted for analysis as outlined above. The relative uptake of the targeted radiotracer in the distal hindlimb was computed by a single operator. The I/NI ratios of activity within the distal hindlimbs were compared using ANOVA, followed by a paired Student $t$ test.

\section{RESULTS}

\section{Validation of Image-Analysis Approach}

To evaluate the accuracy of our quantitative image-analysis approach, the image-derived I/NI activity ratios from both proximal and distal hindlimbs were plotted against corrected ratios derived by GWC of tissue. This initial analysis demonstrated a poor correlation $\left(R^{2}=0.05\right)$ between the quantitative image-derived results and GWC (Fig. 2A). However, we identified animals $(n=5)$ in which there was evidence of significant scatter into the proximal segments from highly

FIGURE 1. Method for analysis of micro-SPECT/CT images. (A) Planes were interactively positioned over lower body of mice to segment micro-CT to generate multiple VOls. (B) Contours of these VOls are illustrated superimposed on representative micro-CT image. (C) $\mathrm{K}$-means clustering-based segmentation was performed to eliminate bones from VOls. (D) This complex irregular object map was applied to registered micro-SPECT images to determine mean counts in each VOI.

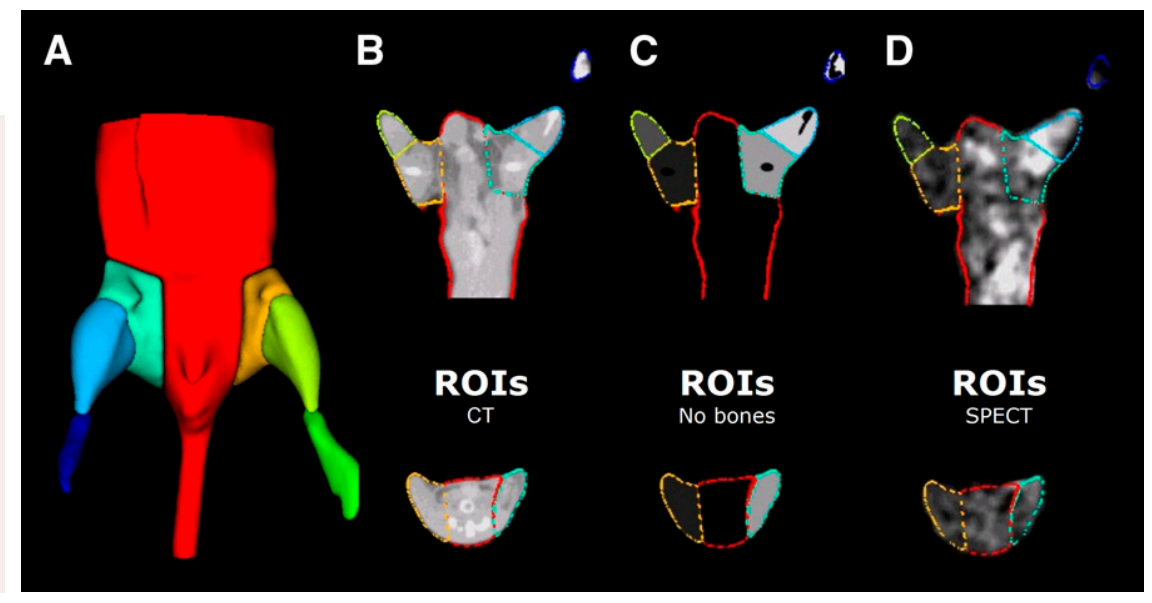




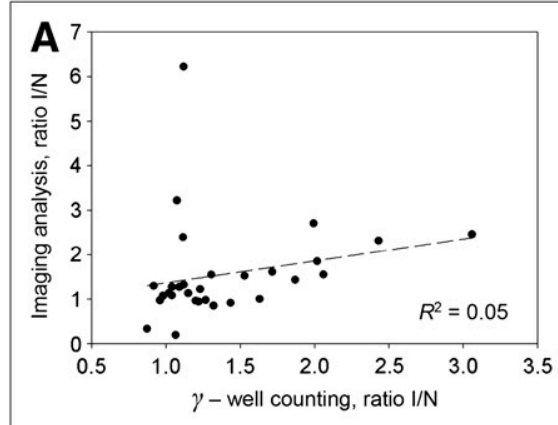

C
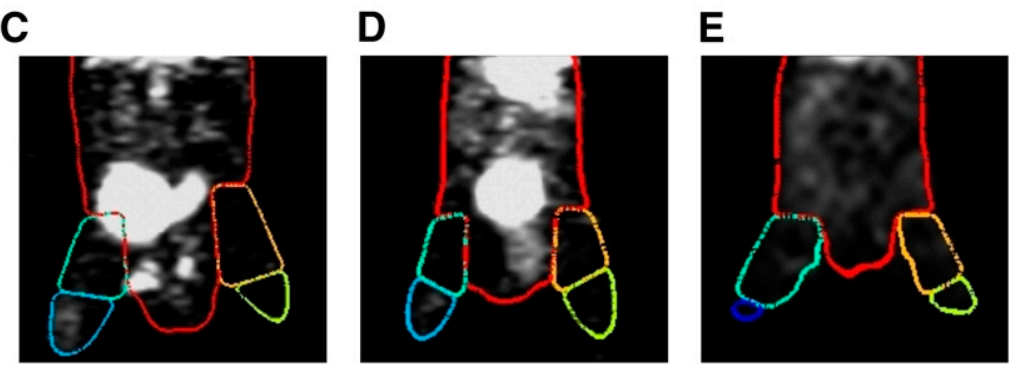

FIGURE 2. Validation of micro-SPECT/ CT image-analysis approach. (A) Correlation between $\mathrm{I} / \mathrm{NI}$ ratios of counts calculated from image analysis and GWC for both proximal and distal regions. Correlation was poor $\left(R^{2}=0.05\right)$ when all proximal and distal regions were included. (B) Correlation coefficient between imaging analysis and GWC improved significantly $\left(R^{2}=\right.$ 0.63) after proximal regions contaminated with scatter from radioactivity in bladder were removed from analysis. Shown are representative $99 \mathrm{mTc}$ NC100692 micro-SPECT/CT images from 3 mice at $7 \mathrm{~d}$ after surgical ligation of right femoral artery with superimposed VOls. (C) Mouse with radioactivity within bladder, which falls within right proximal VOI, resulting in overestimation of counts in ischemic (right) relative to nonischemic (left) proximal leg. (D) Second mouse with bladder located centrally in body and no significant contamination of proximal hindlimb VOls. (E) Images from third mouse after removal of radioactive urine from bladder by needle aspiration immediately before SPECT acquisition.

radioactive urine within the animal's bladder. This scatter resulted in a variable overestimation of counts in the proximal hindlimb from image analysis. After excluding from the analysis those mice with clear contamination of the proximal VOIs, the correlation coefficient between the quantitative image analysis and GWC was excellent (Fig. 2B), with a significantly improved correlation coefficient $\left(R^{2}=0.63\right)$. Figure 2C shows a representative micro-SPECT image of an animal with radioactivity within the bladder that was positioned toward the proximal limb and encroaching into the proximal VOI of the ischemic hindlimb. In most of the animals, the bladder did not affect the quantitative image analysis of proximal sections. Figure 2D shows a microSPECT image of the animal with the radioactive bladder located centrally with no appreciable effect on image-derived activity from the proximal VOIs. To eliminate the scatter of activity within the bladder into the proximal VOIs, we aspirated the urine from the bladder using a 30-gauge needle immediately before the micro-SPECT scan. Figure $2 \mathrm{E}$ shows a representative micro-SPECT image from a mouse after removal of the radioactive urine immediately before initiation of the micro-SPECT acquisition. Although this approach can potentially improve image quantification of proximal VOIs, this intervention may not be applicable for repeated serial imaging of animals over a prolonged period. In most of the mice analyzed, the bladder did not affect the image analysis.

The specificity of ${ }^{99 m}$ Tc-NC100692 uptake toward $\alpha_{\mathrm{v}}$ integrin was validated by the use of ${ }^{99 m}$ Tc-AH-111744-01, a negative-control radiotracer containing a scrambled peptide. GWC analysis of muscles from the distal leg demonstrated a significant increase in the I/NI ratios in ${ }^{99 \mathrm{~m}} \mathrm{Tc}-\mathrm{NC} 100692-$ injected animals $(2.10 \pm 0.29, n=5)$ relative to the negativecontrol group (1.20 $\pm 0.28, n=5, P<0.05)$.

Our previous studies confirmed that the angiogenic response is seen primarily in the muscles of the distal leg (12). Thus, the subsequent validation studies were performed using results obtained from only the distal hindlimb. The accuracy of our 3D volumetric and traditional 2D approaches were evaluated by comparing the calculated I/NI ratios from distal 3D VOIs and 2D ROIs with the results derived from corrected GWC ratios. The results for the distal hindlimb are summarized in Figure 3. The quantitative image analysis was highly correlated with $\gamma$-counting, yielding a high correlation coefficient $\left(R^{2}=0.78\right)$ for both $3 \mathrm{D} \mathrm{VOI}$ and $2 \mathrm{D}$ ROI analysis. The residuals plot for both methods confirmed the quality of the fit. Although the correlation coefficients obtained using our new 3D volumetric and traditional 2D analysis method are similar, there was a significant underestimation of counts quantified using 2D ROIs (Fig. 3C). The intraobserver (Fig. 4A) and interobserver (Fig. 4B) variabilities of our 3D volumetric analysis were excellent, as reflected by the high correlation coefficients $\left(R^{2}=0.997\right)$.

\section{Serial Micro-SPECT/CT of Peripheral Angiogenesis}

Micro-SPECT/CT images at $60 \mathrm{~min}$ after injection of 99mTc-NC100692 were of excellent quality and allowed for quantitative analysis of radiotracer retention in the ischemic distal limb. Figure 5A shows representative micro-SPECT/ CT images recorded in wild-type and eNOS-knockout mice at baseline, $7 \mathrm{~d}$, and $4 \mathrm{wk}$ after femoral artery resection. Differences in the image-derived ratios of radiotracer retention $(\mathrm{I} / \mathrm{NI})$ are shown in Figure 5B. A significant $(P<0.05)$ increase in ${ }^{99 \mathrm{~m}} \mathrm{Tc}-\mathrm{NC} 100692$ relative retention was observed 

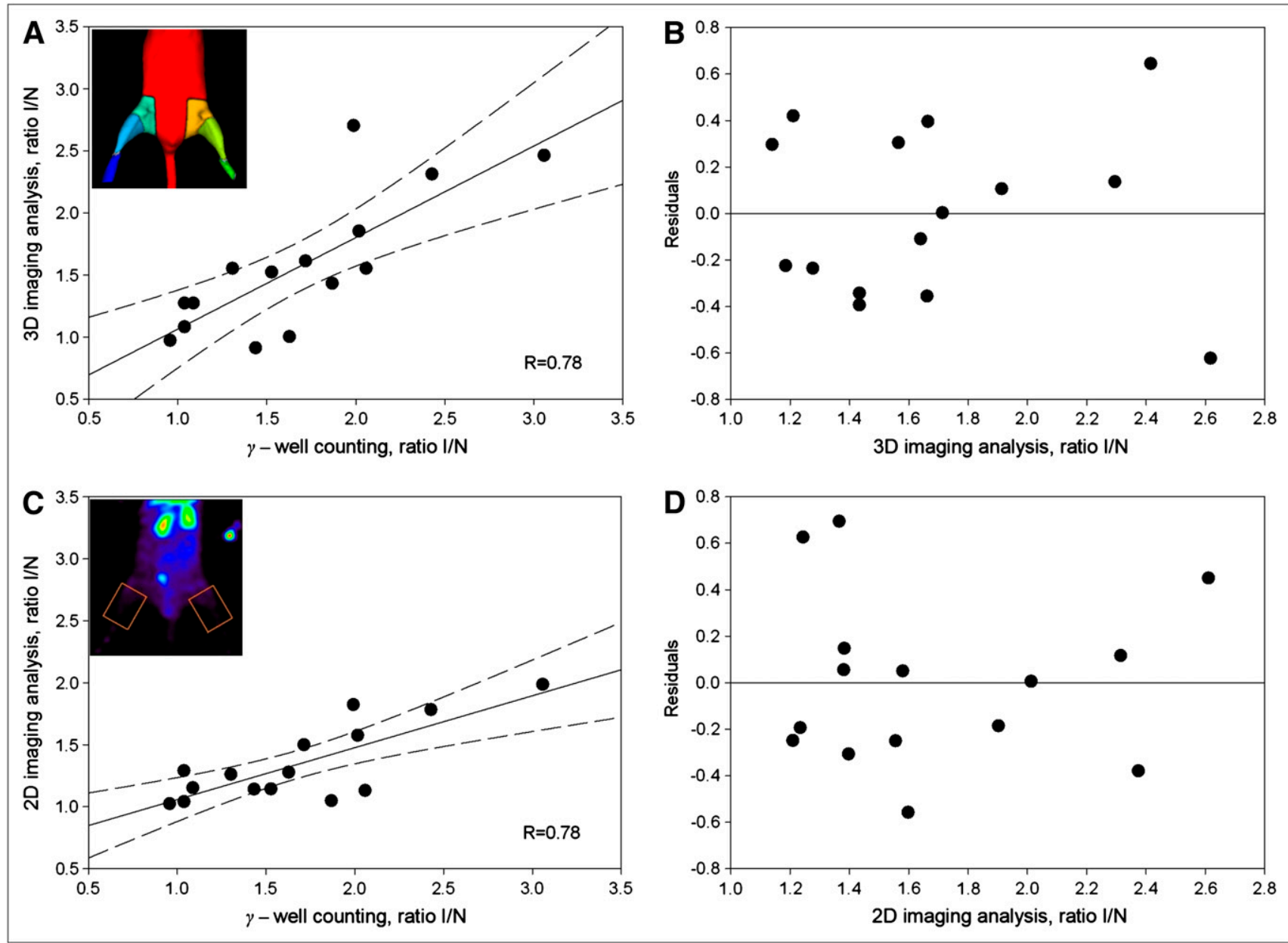

FIGURE 3. Validation of analysis of distal hindlimb. There was good correlation $(R=0.78)$ between ischemic-to-nonischemic counts ratio calculated from $3 \mathrm{D}$ volumetric analysis of micro-SPECT/CT images $(\mathrm{A})$ and $2 \mathrm{D}$ analysis of maximum-projection images (C) relative to GWC for distal hindlimb. Linear correlation (solid line) and 95\% confidence intervals (dashed line) are shown (B and $D)$. Quality of fit was confirmed by residuals plot for micro-SPECT/CT image (B) and 2D maximum-projection image (D) analysis approaches.

in both wild-type (3.81 \pm 0.60$)$ and eNOS $-/-$ mice $(2.85 \pm$ $0.43)$ at $7 \mathrm{~d}$ from the onset of ischemia. Moreover, there was a significant $(\sim 25 \%)$ decrease in relative radiotracer retention in the ischemic distal hindlimb of eNOS-knockout mice, compared with wild-type animals $(P<0.05)$, suggesting an impairment of the angiogenic response at this time point. At baseline and $4 \mathrm{wk}$ after femoral artery resection, there were no significant differences in relative ${ }^{99 \mathrm{~m}} \mathrm{Tc}-\mathrm{NC} 100692$ retention in the ischemic distal hindlimb between the wild-type (baseline, $1.47 \pm 0.27 ; 4 \mathrm{wk}, 2.15 \pm 0.79$ ) and eNOS $-/-$ mice (baseline, $1.25 \pm 0.58 ; 4 \mathrm{wk}, 2.14 \pm 0.29, P=$ not statistically significant).

\section{DISCUSSION}

The experimental evaluation of spatial and temporal changes in peripheral angiogenesis is traditionally done using highly invasive techniques that require the collection and often the destruction of tissue samples, which prohibits serial monitoring of this biologic process in living animals.
We demonstrated the feasibility of serial ${ }^{99 \mathrm{~m}} \mathrm{Tc}-\mathrm{NC} 100692$ micro-SPECT/CT for the noninvasive monitoring of ischemiainduced angiogenesis in vivo using an established murine model of hindlimb ischemia. Applying this targeted-imaging approach, we were also able to confirm the previously observed early impairment of angiogenesis after ischemic injury in transgenic mice in which eNOS expression was reduced. To specifically target proliferating angiogenic vessels, we used a ${ }^{99 \mathrm{~m}} \mathrm{Tc}$-labeled RGD peptide targeted to $\alpha_{\mathrm{v}}$ integrins known to be activated during the initiation of the angiogenic process. The specificity of the binding of the RGD peptide for activated endothelial cells was previously demonstrated using a fluorescein-labeled negative-control compound containing a scrambled peptide (5). In this study, we have confirmed the specificity of the RGD peptide using a ${ }^{99 \mathrm{~m}} \mathrm{Tc}-$ labeled negative-control compound (AH-111744-01) containing a scrambled peptide.

The novel noninvasive imaging approach for the quantitative serial assessment of angiogenesis in mice became 

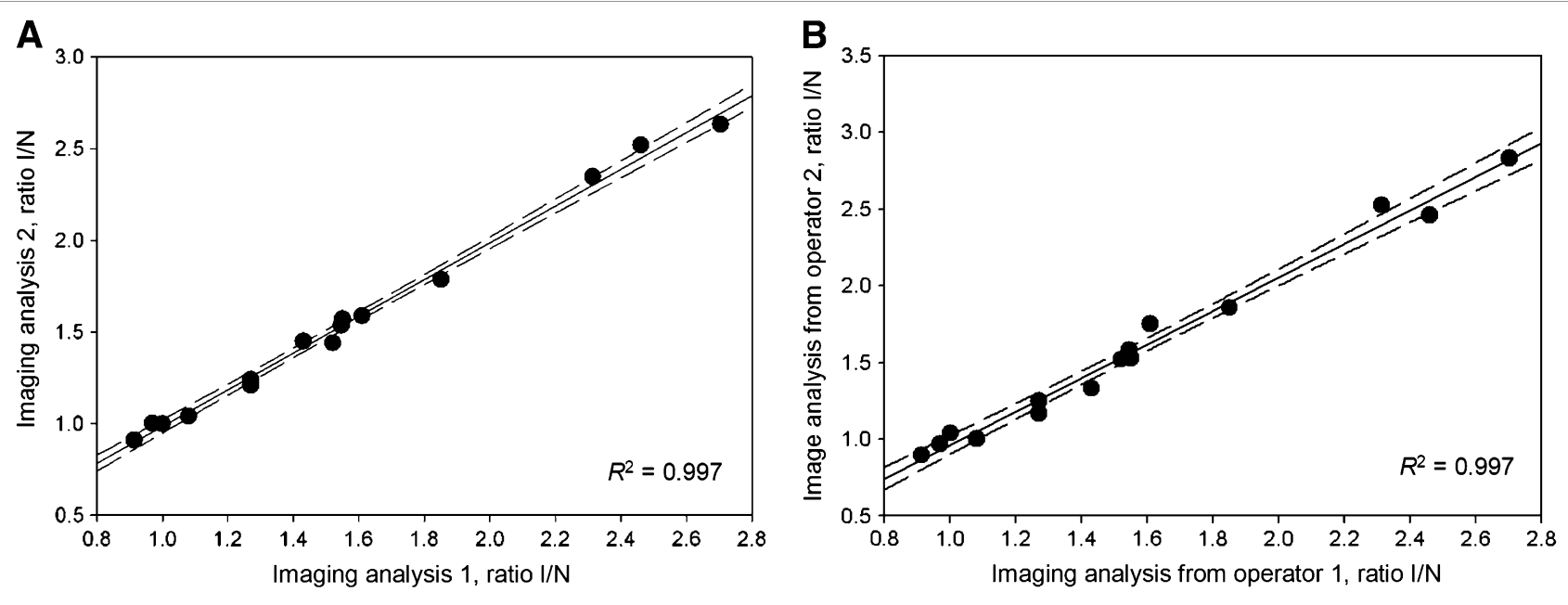

FIGURE 4. Reproducibility of image quantification. Intraobserver (A) and interobserver (B) reproducibility of micro-SPECT/CT image analysis were excellent.

possible with the development of targeted radiotracers such as ${ }^{99 \mathrm{~m}} \mathrm{Tc}-\mathrm{NC} 100692$ and the development of dedicated small-animal imaging systems. Among different imaging modalities, both PET and SPECT are currently highly suited for molecular targeted imaging of biologic processes, including angiogenesis, because of the relatively high sensitivity and capability for 3D imaging and quantification. The advantages and disadvantages of these 2 modalities have been described in detail in previously published reviews (1316). Briefly, PET provides higher sensitivity and more established attenuation-correction algorithms. On the other hand, SPECT generally provides a better spatial resolution than PET for small-animal imaging and allows for the use of multiple isotopes simultaneously. The recently introduced hybrid imaging systems combining anatomic (CT, MRI) with functional (PET, SPECT) imaging allow for the more accurate definition of ROIs or VOIs and partial-volume and attenuation corrections, which are important steps in standardizing the quantification of small-animal nuclear images.

In this study, the lower-resolution, high-sensitivity microSPECT images were reconstructed and fused with the registered high-resolution anatomic micro-CT images for

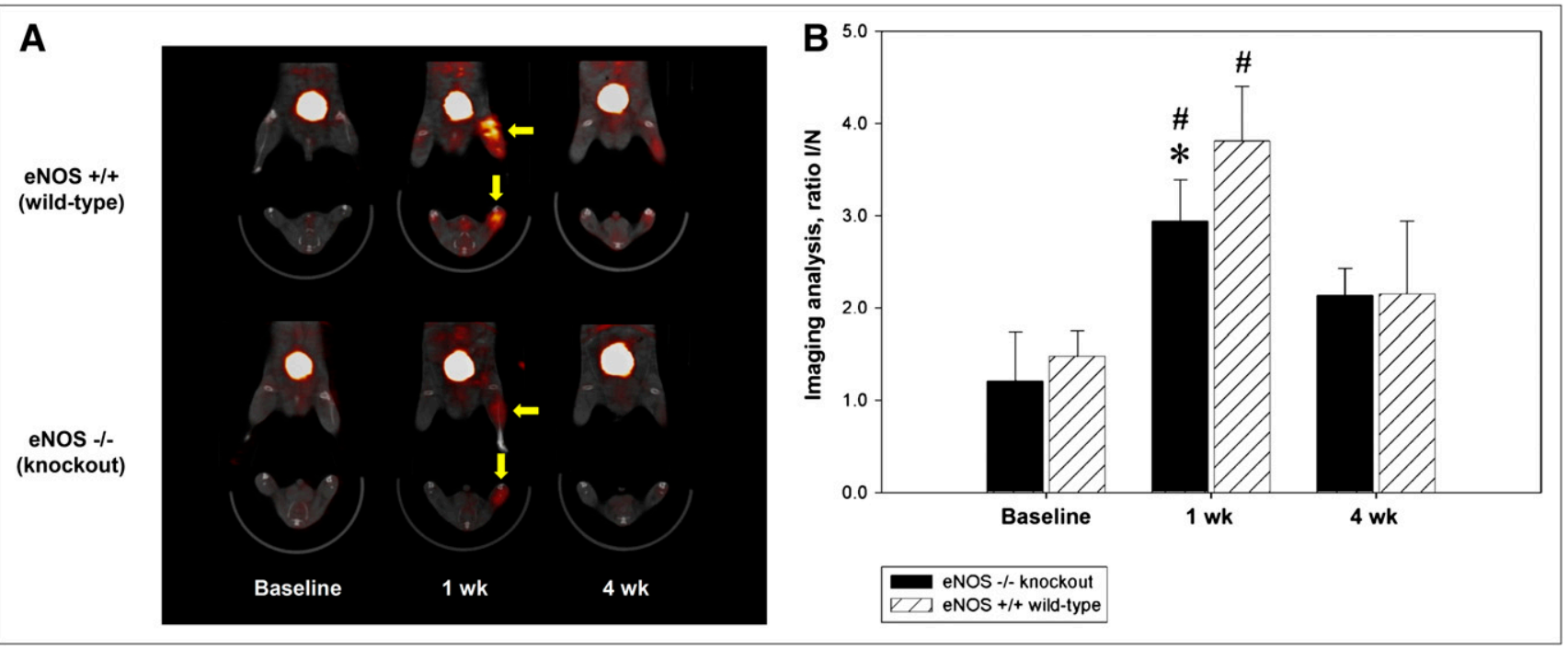

FIGURE 5. Analysis of wild-type and eNOS-knockout mice. (A) Representative micro-SPECT/CT images of eNOS $+/+$ and eNOS $-/-$ mice injected with ${ }^{99 m}$ Tc-NC100692 at baseline, $7 \mathrm{~d}$, and 4 wk after right femoral artery ligation. Yellow arrows

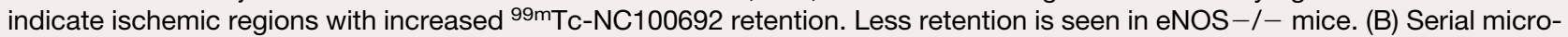
SPECT/CT images were analyzed and I/NI ${ }^{99 m}$ Tc-NC100692 activity ratios calculated. There was significant $(P<0.05)$ increase of $99 \mathrm{mTc}-\mathrm{NC} 100692$ retention in ischemic leg at $7 \mathrm{~d}$ after surgery in both groups. However, there was significantly less retention in eNOS $-/-$ mice at $7 \mathrm{~d}$ than in wild-type mice. ${ }^{\star} P<0.05$ vs. wild-type. $\# P<0.05$ vs. baseline. 
quantitative analysis. The analysis was based on CT segmentation of murine hindlimbs, definition of 3D VOIs, and calculation of the I/NI activity ratio from the micro-SPECT images. Our image-derived quantitative analysis of relative radiotracer retention was validated with GWC of tissue samples, an established gold-standard technique. We found a strong positive correlation $\left(R^{2}=0.78\right)$ between relative (I/NI) retention of radiotracer assessed by image analysis and the GWC in sections from the lower hindlimb distal to the femoral occlusion. We compared our semiautomated $3 \mathrm{D}$ volumetric approach with more traditional manually defined 2D ROIs on planar equivalent maximum-projection nuclear images. Although the comparison of these 2 approaches with GWC analysis resulted in similar correlation coefficients, we observed that 2D analysis tends to underestimate the counts derived from radiotracer uptake (Fig. $3 \mathrm{C})$. This phenomenon was observed and described in our previous studies (5). We hypothesized that this underestimation could be partially due to the selection of inappropriate ROIs, and we proposed that the images could be more accurately quantified using hybrid micro-SPECT/CT, allowing for anatomic localization and definition of 3D VOIs. Indeed, our 3D volumetric approach significantly improved the counts derived from ischemic hindlimb (Fig. 3A). Manual definition of ROIs or VOIs by the operator introduces variability and a potential bias to the analysis. In contrast, our approach is based strictly on definition of VOIs using anatomic landmarks including lower-extremity joints and skeletal structures, which resulted in an excellent inter- and intraobserver reproducibility, as reflected by the high correlation coefficients.

The quantitative hybrid micro-SPECT/CT approach allows for the noninvasive analysis of both proximal and distal murine hindlimbs, although we have identified potential limitations related to intense bladder activity, which may reduce the reliability of the analysis from the proximal hindlimb located above the site of ligation of the femoral artery. The scatter of activity from the bladder resulted in a variable overestimation of counts in the proximal hindlimb estimated from the micro-SPECT/CT images. Although the aspiration of radioactivity from the bladder improved our analysis of proximal sections, this solution may not be ideal for serial imaging of the same animal over prolonged periods because of the potential risk for infection. Despite this limitation for estimation of angiogenesis in proximal hindlimb segments, there was an almost-perfect observer-independent reproducibility of the image analysis, which strongly correlated with the GWC in the distal segments. This noninvasive micro-SPECT/CT approach holds great promise for the assessment of both spatial and temporal changes in peripheral angiogenesis in animals after ischemic injury and for the evaluation of therapeutic intervention.

To further demonstrate the applicability of this quantitative targeted $\alpha_{\mathrm{v}}$ integrin micro-SPECT/CT approach for the in vivo serial assessment of angiogenesis, we evaluated 2 strains of mice with known differences in their angiogenic response after ischemic injury. Serial micro-SPECT/CT images were acquired in eNOS-knockout mice lacking endothelial nitric oxide, which is a crucial mediator of angiogenesis. The mice lacking eNOS have been previously shown to have decreased peripheral angiogenesis in response to ischemia and significantly impaired flow recovery in the distal hindlimb as determined using highly invasive techniques including deep-penetrating laser Doppler imaging and histologic analysis (1). In the current study, eNOSknockout and control mice were serially imaged at baseline and at 1 and $4 \mathrm{wk}$ after surgical resection of the right femoral artery that resulted in severe ischemia using the same imaging protocol as in validation experiments. As expected, we observed a significant increase of ${ }^{99 \mathrm{~m}} \mathrm{Tc}-\mathrm{NC} 100692$ retention within ischemic distal limbs in both animal groups at $1 \mathrm{wk}$ after ligation, which normalized by $4 \mathrm{wk}$. The analysis of serial micro-SPECT/CT images revealed that there is a statistically significant $(P<0.05)$ reduction in relative ${ }^{99 \mathrm{~m}} \mathrm{Tc}-\mathrm{NC} 100692$ retention in eNOS knockouts at $1 \mathrm{wk}$ from the surgery, suggesting impaired peripheral angiogenesis in these knockout mice. These differences between the groups normalized at $4 \mathrm{wk}$ after ligation. The observed differences between the groups obtained using noninvasive quantitative analysis of targeted micro-SPECT/CT 99mTc-NC100692 imaging were in good agreement with those obtained using more invasive techniques previously published by our group (1). These invasive techniques included immunohistochemistry of microscopic sections, Western blotting, and Doppler laser to assess perfusion. All of these approaches required the irreversible destruction of a biopsied sample or significant surgical insult, making serial evaluations nonfeasible.

\section{CONCLUSION}

We have developed a reproducible and accurate approach for the semiautomated analysis of micro-SPECT/CT images for the quantitative evaluation of angiogenesis, which is applicable to murine models of ischemia-induced peripheral angiogenesis. The analysis approach has been carefully validated and is now freely available online (http://www. bioimagesuite.org/) as a user-friendly image-analysis tool. This hybrid targeted-imaging approach would be potentially applicable for the evaluation of other biologic processes in animals, using a wide range of potential targeted radiotracers for micro-SPECT/CT or small-animal PET/CT. Although this image-analysis approach was designed to analyze murine micro-SPECT/CT images, the approach also holds promise for future clinical translational studies involving the in vivo imaging of angiogenesis and other biologic processes in patients with peripheral arterial disease.

\section{ACKNOWLEDGMENTS}

This work was supported in part by the NIH, under grants R01HL065662 and R01EB006494, and Juvenile Diabetes Research Foundation grant 10-2006-726. 


\section{REFERENCES}

1. Yu J, deMuinck ED, Zhuang Z, et al. Endothelial nitric oxide synthase is critical for ischemic remodeling, mural cell recruitment, and blood flow reserve. Proc Natl Acad Sci USA. 2005;102:10999-11004.

2. Cai W, Chen K, Mohamedali KA, et al. PET of vascular endothelial growth factor receptor expression. J Nucl Med. 2006;47:2048-2056.

3. Chen X, Sievers E, Hou Y, et al. Integrin $\alpha_{\mathrm{v}} \beta_{3}$-targeted imaging of lung cancer. Neoplasia. 2005;7:271-279.

4. Collingridge DR, Carroll VA, Glaser M, et al. The development of $\left[{ }^{124} I\right]$ iodinatedVG76e: a novel tracer for imaging vascular endothelial growth factor in vivo using positron emission tomography. Cancer Res. 2002;62:5912-5919.

5. Hua J, Dobrucki LW, Sadeghi MM, et al. Noninvasive imaging of angiogenesis with a ${ }^{99 m}$ Tc-labeled peptide targeted at $\alpha_{\mathrm{v}} \beta_{3}$ integrin after murine hindlimb ischemia. Circulation. 2005;111:3255-3260.

6. Li S, Peck-Radosavljevic M, Kienast O, et al. Iodine-123-vascular endothelial growth factor-165 ( $\left.{ }^{123} \mathrm{I}-\mathrm{VEGF} 165\right)$ : biodistribution, safety and radiation dosimetry in patients with pancreatic carcinoma. Q J Nucl Med Mol Imaging. 2004; 48:198-206.

7. Lu E, Wagner WR, Schellenberger U, et al. Targeted in vivo labeling of receptors for vascular endothelial growth factor: approach to identification of ischemic tissue. Circulation. 2003;108:97-103.
8. Bach-Gansmo T, Danielsson R, Saracco A, et al. Integrin receptor imaging of breast cancer: a proof-of-concept study to evaluate ${ }^{99 \mathrm{~m}} \mathrm{Tc}-\mathrm{NC} 100692 . \mathrm{J} \mathrm{Nucl}$ Med. 2006;47:1434-1439.

9. Edwards D, Jones P, Haramis H, et al. ${ }^{99 \mathrm{~m} T c-N C 100692: ~ a ~ t r a c e r ~ f o r ~ i m a g i n g ~}$ vitronectin receptors associated with angiogenesis - a preclinical investigation. Nucl Med Biol. 2008;35:365-375.

10. Indrevoll B, Kindberg GM, Solbakken M, et al. NC-100717: a versatile RGD peptide scaffold for angiogenesis imaging. Bioorg Med Chem Lett. 2006;16: 6190-6193.

11. Kenny LM, Coombes RC, Oulie I, et al. Phase I trial of the positron-emitting Arg-Gly-Asp (RGD) peptide radioligand ${ }^{18} \mathrm{~F}-\mathrm{AH} 111585$ in breast cancer patients. J Nucl Med. 2008;49:879-886.

12. Hua J, Bourke B, Song J, et al. Noninvasive detection of angiogenesis, with a technetium- $99 \mathrm{~m}$ labeled peptide targeted at alpha $_{\mathrm{v}}$ beta $_{3}$ integrin following hindlimb ischemia [abstract]. J Am Coll Cardiol. 2004;43:25A.

13. Dobrucki LW, Sinusas AJ. Molecular imaging: a new approach to nuclear cardiology. Q J Nucl Med Mol Imaging. 2005;49:106-115.

14. Dobrucki LW, Sinusas AJ. Molecular cardiovascular imaging. Curr Cardiol Rep. 2005;7:130-135.

15. Dobrucki LW, Sinusas AJ. Cardiovascular molecular imaging. Semin Nucl Med. 2005;35:73-81.

16. Sinusas AJ. Imaging of angiogenesis. J Nucl Cardiol. 2004;11:617-633. 